\title{
Comprehensive planning and the role of SDSS in flood disaster management in Malaysia
}

\begin{abstract}
Purpose - To present a comprehensive flood management plan for Malaysia, the various planning stages and the proponents of the plan. It is also to expound and highlight the importance of spatial information technology in the strategy and to outline the critical decision-making at various levels of the plan. Design/methodology/approach - A review of flood disaster management aimed at providing an insight into the strategies for a comprehensive flood disaster management for Malaysia. Discussion of the framework of a spatial decision support system (SDSS) and its role in decision-making in a comprehensive disaster management plan. Findings - Provides information about a proposed comprehensive disaster management program for Malaysia and highlight the role of SDSS in improving decision-making. It recognizes the strength of SDSS in the collection and processing of information to speed up communication between the proponents of the disaster management program. A well-design SDSS for flood disaster management should present a balance among capabilities of dialog, data and modeling. Research limitations/implications - The study has outlined the links and components of SDSS and not its development processes; this may limit the used of this paper in in-depth study of the development if SDSS. Some source for detail study of the development of SDSS have, however, been cited. Practical implications - This paper is a very useful source of information about the preparation of a comprehensive disaster management program. It also sheds light on the role of SDSS in improving and speeding up communication between the various proponents of the program. Researcher and students will fine, it provides general guidelines and framework for disaster planning and management. Originality/value - This paper fulfills flood disaster study need for developing a comprehensive disaster management program. It presents the framework of SDSS, the interrelationship between their various components and how they play a role in decisionmaking.
\end{abstract}

